\title{
effects of omega-3 PUFA (ALA) on WT1 gene expression in pancreatic cancer cell line (MIA PaCa-2)
}

\author{
Babak Rahmani (1) \\ Dariush Hamedi Asl (1) \\ Taghi Naserpour Farivar (2) \\ Mehdi Azad (3) \\ Mehdi Sahmani (4) \\ Nematollah Gheibi (2) \\ 1-Department of Molecular Medicine, Faculty of Medical Sciences, Qazvin University of \\ Medical Sciences, Qazvin, Iran \\ 2-Cellular and Molecular Research Centre, Qazvin University of Medical Sciences, Qazvin, \\ Iran \\ 3-Department of Medical laboratory sciences, Faculty of Allied Medicine, Qazvin University \\ of Medical Sciences, Qazvin, Iran \\ 4-Department of Clinical Biochemistry and Genetic, Faculty of Medicine, Qazvin University \\ of Medical Sciences, Qazvin, Iran \\ Corresponding authors: \\ Nematollah Gheibi, PhD \\ Cellular and Molecular Research Center, Qazvin University of Medical Sciences, Qazvin, \\ Iran \\ Phone: +982833375160 \\ Email: ngheibi@qums.ac.ir ; gheibi n@yahoo.com \\ Mehdi Sahmani, PhD \\ Mailing address: Dr. M. Sahmani; Department of Clinical Biochemistry and Genetic, Faculty \\ of Medicine, Qazvin University of Medical Sciences, Qazvin - Iran \\ Phone: +982833328213 \\ Email:m.sahmani@gmail.com
}

\begin{abstract}
Background: Cancer starts by cells acquiring a number of characteristic alterations especially abnormal cell growth. These properties reflect faults in signalling pathways in cancer cells. Wilms' tumor 1(WT1) acts as a tumor suppressor by negative reg-ulation of WNT/beta-catenin signaling pathway. The role of WT1 in many cancers has been studied.Aims: To evaluate the WT1 gene expression alterations in response to different concentrations of omega-3 PUFA alpha linolenic acid (ALA) in pancreatic cancer cell line (MIA PaCa-2) in a time dependent manner.Methods: MiaPaca-2cell line was cultured in monolay-ers. After that began the cells treatment with omega3 fatty acid (ALA) using different concentrations of 25, 50, 100, 250, 500 and $1000 \mu \mathrm{M}$ for 24, 48 and 72 hours. The RNA was extracted from both control (untreated) and treated cells. Viability was checked by MTT as-say and WT1 expression was evaluated by RT-PCR.

Results: Real-time RT-PCR analysis showed that the level of WT1 mRNA, was decreased after 24, 48, 72 hours treatment with omega-3 fatty acid (ALA) for 100, 250, 500 and $1000 \mu \mathrm{M}$
\end{abstract}


concentrations but not 25 and $50 \mu \mathrm{M}$. Data obtained from MTT revealed an-tiproliferative effects of omega-3 fatty acid (ALA) for $100-1000 \mu \mathrm{M}$ concentrations but not 25 and $50 \mu \mathrm{M}$.

Conclusion: Our findings indicated that the cell vi-ability and level of WT1 mRNA was decreased dur-ing Omega 3 ALA Treatment. So, it a potential role of Omega 3 in prevention or as a supplementary for pancreatic cancer treatment has been suggested

.Key words: omega-3 PUFA (ALA), pancreatic can-cer, MIA PaCa-2, WT1.

Please cite this article as: Herati E. et al. The effects of omega-3 PUFA (ALA) on WT1 gene expression in pancreatic cancer cell line (MIA PaCa-2). 2018;16(2):275-281. World Family Medicine.

DOI: 10.5742/MEWFM.2018.93287 